\title{
ON RAMIFIED RIEMANN DOMAINS
}

\author{
YOSHIO TOGARI
}

Let $\varphi$ be a holomorphic mapping of an $n$-dimensional analytic space $E$ into $C^{n}$. If $\varphi$ is non-degenerate at every point of $E$, we call the pair $(E, \varphi)$ a Riemann domain. The notion of a Riemann domain is a generalization of the notion of a concrete Riemann surface. A Riemann domain $(E, \varphi)$ is said to be unramified if $\varphi$ is a local homeomorphism, and to be ramified if otherwise.

In the classical theory of functions of several complex variables, most considerations have been done only on unramified Riemann domains. If we permit (holomorphic-) algebroidal functional elements in the analytic prolongation, there appears the ramified Riemann domain. Some of the classical results concerning unramified Rimann domains do not hold for ramified Riemann domains, and we know very little to what extent the results in the unramified case can be generalized to the ramified case. The purpose of the present paper is to investigate some properties of ramified Riemann domains.

In $\$ 1$ we recall the notion of analytic spaces and introduce some terminologies for the later use. In $\$ 2$ a Riemann domain and its boundary are defined, and their properties are stated. After these preparations we consider in $\S 3$ the holomorphic prolongations of holomorphic functions on a Riemann domain. As in the unramified case, for a holomorphic function $f$ on a Riemann domain we can construct canonically the Riemann domain of the maximal holomorphic prolongation of $f$ or the existence domain of $f$. Thus we arrive at the notion of the domain of holomorphy. It is well known that in the unramified case a domain of holomorphy is characterized by its holomorphic convexity. However in general cases such characterization is impossible." So we are led to consider a certain condition which is strictly weaker than the holomorphic convexity, and to prove its sufficiency for a Riemann domain to be a domain of holomorphy; this forms a main result of the present paper and is given in $\$ 4$ Theorem 4 .

Received March 5, 1958.

1) Cf. H. Grauert and R. Remmert [8]. 


\section{$\S 1$. Preliminaries}

1. In an open set $U$ in the complex affine space $C^{s}$ of dimension $s, a$ subset $V \subset U$ is an analytic subset of $U$ if $V$ is closed in $U$ and if at each point $x \in V, V$ is defined as the common zeros of holomorphic functions at $x^{2}{ }^{2}$ Let $\mathfrak{D}_{x}(U)$ be the ring of all germs of holomorphic functions at $x \in U$. For a point $x \in V$, let $\mathfrak{D}_{x}(V)$ be the quotient ring $\mathfrak{D}_{x}(U) / \Im_{x}(V)$, where $\Im_{x}(V)$ is the ideal of $\mathfrak{D}_{x}(U)$ consisting of all germs which are identically zero on $V$. If, for every point $x \in V, \mathfrak{D}_{x}(V)$ is an integrally closed integral domain, $V$ is said to be a normal analytic subset of $U$. For two normal analytic subsets $V$, $V^{\prime}$, a continuous mapping $\lambda: V \rightarrow V^{\prime}$ is called a holomorphic mapping, if the assignement $\lambda^{*}: f \rightarrow f \circ \lambda\left(f \in D_{\lambda(x)}\left(V^{\prime}\right)\right)$ defines a ring-homomorphism of $\mathfrak{D}_{\lambda(x)}\left(V^{\prime}\right)$ into $\mathfrak{D}_{x}(V)$ for every $x \in V$. Further if $\lambda$ is a homeomorphism of $V$ onto $V^{\prime}$, $\lambda$ is called a holomorphic isomorphism between $V$ and $V^{\prime}$.

An analytic space is a connected Hausdorff space $E$ such that $E$ has an open covering $\left\{\Delta_{i}\right\}$ where each $\Delta_{i}$ is homeomorphic by $\lambda_{i}$ to a normal analytic subset $V_{i}$ of an open set in some $C^{s_{i}}$ and, for any pair $\Delta_{i}, \Delta_{j}\left(\Delta_{i} \cap \Delta_{j} \neq \phi\right)$, $\lambda_{j} \circ \lambda_{z}^{-1}$ is a holomorphic isomorphism between $\lambda_{i}\left(\Delta_{i} \cap \Delta_{j}\right)$ and $\lambda_{j}\left(\Delta_{i} \cap \Delta_{j}\right)^{3}{ }^{3}$

For every point $x$ of an analytic space $E$, put $\mathfrak{D}_{x}(E)=\left\{f \circ \lambda_{i} / f \in \mathfrak{D}_{\lambda_{i}(x)}\left(V_{i}\right)\right.$, where $V_{i}$ is a normal analytic subset homeomorphic to $\Delta_{i}$ containing $x$. There may exist many such $\Delta_{i}$, but, according to the definition, $\mathscr{D}_{x}(E)$ is determined uniquely independent of the special choice of $\Delta_{i}$. A point $x \in E$ is called uniformisable if $\mathfrak{D}_{x}(E)$ is isomorphic to the ring of all convergent power series in $n$ complex variables for some $n$. If all points of $E$ are uniformisable, $E$ is nothing but a complex manifold. It is known that every point of $E$ possesses a fundamental system of neighborhoods such that the intersection of each neighborhood and the set of uniformizable points in $E$ is connected." We define the dimension of an analytic space as the dimension of the (connected) complex manifold composed of all uniformizable points in it. Also, for any point $x \in E, D_{x}(E)$ is canonically isomorphic to the ring of all the germs at $x$

2) For details in this section, see H. Cartan [4], [5].

3) This definition of the analytic space is due to H. Cartan ("espace analytique générale" in [4], [5]). Recently H. Grauert and R. Remmert have noticed in [9] that this is equivalent to the definition of "komplexer Raum" in [7].

4) Cf. H. Cartan [4] XII and XIII. 
which are continuous and aré holomorphic at each uniformizable point." Therefore, the holomorphic function in an analytic space may be defined as follows: a complex valued function $f$ on an open set $\Delta$ in $E$ is holomorphic on $\Delta$ if $f$ is continuous on $\Delta$ and holomorphic at all uniformizable points in $\Delta$. Then the set of all germs of holomorphic functions at $x \in E$ coincides with $\mathfrak{O}_{x}(E)$. We can define the notion of a holomorphic mapping, a holomorphic isomorphism and an analytic subset for analytic spaces. Here it should be remarked that the whole of non-uniformizable points in an analytic space $E$ of dimension $n$ forms an at most $(n-2)$-dimensional analytic subset of $E^{5}$ ) In particular each 1-dimensional analytic space is a complex manifold. Analytic spaces of dimension $>1$ have in general so called algebroidal singularities. For holomorphic functions on an analytic space, the theorem of identity and the maximum principle hold.

2. Let

$$
P((z), w)=\left(w-w_{0}\right)^{p}+a_{1}((z))\left(w-w_{0}\right)^{p-1}+\ldots+a_{p}((z))
$$

be an irreducible distinguished pseudo-polynomial in the complex variable $w$, at the center $\left(\left(z_{0},\right) w_{0}\right)$, with coefficients holomorphic at $\left(z_{0}\right) \in C^{n}$. Suppose that all $a_{i}((z))$ are holomorphic on a neighborhood $H$ of $\left(z_{0}\right)$. Let $V$ be the analytic subset of $H \times C$ defined by $P((z), w)=0$. Then $V$ determines a space $\tilde{V}$ consisting of all irreducible components $V_{((z), w)}^{i}$ of the germ of $V$ at $((z), w)$, when $((z), w)$ runs over $V$. Now we shall introduce a topology on $\widetilde{V}$ as follows. A point $V_{((z), u)}^{i}$ of $\tilde{V}$ can be represented as an analytic subset $W$ of $N \times C$ for every sufficiently small neighborhood $N$ of $(z) \in C^{n}$, and such $W$ determines a subset of $\tilde{V}$. A base of neighborhoods of $V_{(i z), w)}^{i}$ for the topology on $\tilde{V}$ is defined by the system of all such subsets. ${ }^{6)}$

Then $\tilde{V}$ is a Hausdorff space and the canonical mappings $\pi^{\prime}: \tilde{V} \rightarrow V, \pi^{\prime \prime}$ : $V \rightarrow H, \pi=\pi^{\prime \prime} \circ \pi^{\prime}: \quad \tilde{V} \rightarrow H$ are continuous and proper. Denote by $\delta((z))$ the discriminant of $P((z), w)$ and by $D$ the analytic subset of $H$ defined by $\delta((z))$ $=0$. For abbreviation we call $D$ the discriminant set of $P$. The mapping $\pi$ induces a local homeomorphism of $\tilde{V}-\pi^{-1}(D)$ onto $H-D$. Hence $\tilde{V}-\pi^{-1}(D)$ is an $n$-dimensional connected complex manifold.

5) Cf. H. Cartan [5] XI $\$ 2$.

6) For this topology, see H. Cartan [4] XII. 
It can be proved that $\widetilde{V}$ is an analytic space and that a continuous function $h$ on an open set $\Delta$ in $\widetilde{V}$ is holomorphic if and only if $h$ is holomorphic on the complex manifold $\pi^{-1}(D) .^{i)} \quad$ Consequently, the structure of $\tilde{V}$ as an analytic space is determined by its topological structure and the canonical mapping $\pi$ : $\tilde{V} \rightarrow H$. We call the pair $(\tilde{V}, \pi)$ the Riemann domain of an algebroidal function $P((z), w)=0$ on $H$. The function $f$ defined by $f\left(V_{((z), w)}^{i}\right)=w$ for each point $V_{((z), u)}^{i} \in \tilde{V}$ is holomorphic on $\tilde{V}$ and satisfies the equality $P(\pi(x), f(x))=0$ at any point $x \in \tilde{V}$. We call $f$ the holomorphic function associated with an algebroidal function $P((z), w)=0$.

\section{§. Riemann domains and boundary points}

1. Let $E$ and $E^{\prime}$ be two analytic spaces. A holomorphic mapping $\lambda$ of $E$ into $E^{\prime}$ is said to be non-degenerate at a point $a \in E$ if $\lambda^{-1}(\lambda(a))$ is a discrete set in $E$.

DeFINITION 1. Let $E$ be an n-dimensional analytic space. If there exists a holomorphic mapping $\varphi$ of $E$ into $C^{n}$, which is non-degenerate at every point in $E$, we call the pair $(E, \varphi)$ an n-dimensional Riemann domain. ${ }^{8)}(E, \varphi)$ is said to be unramified if $\varphi$ is a local homeomorphism, and to be ramified if otherwise.

Two Riemann domains $(E, \varphi),\left(E^{\prime}, c^{\prime}\right)$ are isomorphic if there exists a holomorphic isomorphism $\lambda$ of $E$ onto $E^{\prime}$ such that $\varphi=\varphi^{\prime} \circ \lambda$.

The Riemann domain $(\widetilde{V}, \pi)$ of an algebroidal function $P((z), w)=0$ in $\S 1,2$ is a Riemann domain in our present sense.

Let $(E, \varphi)$ be an $n$-dimensional Riemann domain. The whole of nonuniformizable points forms an analytic subset $K$ of $E$ of dimension $\leqq n-2$. In the complex manifold $E-K$, the set of all points at which the Jacobian of $c$ vanishes, is a purely $(n-1)$-dimensional analytic subset $\mathrm{A}^{\prime}$ of $E-K$. By the prolongation theorem of analytic subset, ${ }^{9)} A^{\prime}$ is prolongable to a purely $(n-1)$ -

7) Cf. H. Cartan [5] VII, VIII and also [4] XIII, XIV.

8) An analytic space $E$ is considered as a Riemann domain if and only if, for every point $a \in E$, there exists a holomorphic mapping (depending on $a$ ) of $E$ into some $C^{k}$, which is non-degenerate at $a$. Especially Stein manifolds or abstract Riemann surfaces are considered always as Riemann domains. Cf. H. Grauert [7].

9) Cf. H. Cartan [5] XII Th. 1. 
dimensional analytic subset $A$ of $E$. Denote by $\varphi_{0}$ the restriction of $\varphi$ to $E_{0}=$ $E-(K \cup A)$. Then $\left(E_{0}, \varphi_{0}\right)$ is an unramified Riemann domain. The set $E_{0}$ lies everywhere dense in $E$ and, for any connected neighborhood $\Delta$ of every point in $E, \Delta \cap E_{0}$ is connected. We call $\left(E_{0}, \varphi_{0}\right)$ the unramified Riemann domain associated with (E., ).

2. Now we shall prove the following

Theorem 1. Let $(E, \varphi)$ be a Riemann domain. Then 1) the space $E$ satisfies the second countability axiom, and 2) for any point $a \in E$, there exist a neighborhood $\Delta$ of $a$ and an irreducible distinguished pseudo-polynomial $P((z), w)$ at the center $((b), 0)(b=\varphi(a))$ with coefficients holomorphic at $(b) \in C^{n}$ such that the Riemann domain $\left(A, c_{1}\right)$ (,$c_{1}$ means the restriction of $\varphi$ to $\left.\Delta\right)$ and the Riemann domain $(\widetilde{V}, \pi)$ of the algebroidal function $P((z), w)=0(\$ 1,2)$ are isomorphic. ${ }^{10}$

Proof. 1) Let $\left(E_{0}, \varsigma_{0}\right)$ be the unramified Riemann domain associated with $(E, \varphi)$. Since $E_{\mathrm{J}}$ is connected, we can show, by the same argument as in the proof of the classical Poincaré-Volterra's theorem, that $c_{0}^{-1}\left(\varphi_{10}(x)\right)$ is a countable subset of $E_{n}$ for any point $x \in E_{n}$. The set $S$ of all the points of $E$ whose image by $\varphi_{0}$ have the rational coordinates, is countable and dense in $E_{0}$, and hence, in $E$. Denote by $H((z), \rho)$ the hypersphere with the radius $\rho$ around $(z) \in C^{n}$ and, for a point $x \in E$, denote by $.1(x, \rho)$ the connected component of $\varphi^{-1}(H(c(x), \rho))$ containing $x$. Then we consider the following countable system $\Xi$ of open sets in $E: \Xi=\{A(x, \rho) / x \in S, \rho:$ positive rational numbers $\}$.

Now let $x$ be a point of an open set $\varrho$ in $E$. Since $E$ is locally compact and $\varphi$ is non-degenerate at $x \in E$, there exists a relatively compact open neighborhood $\Delta \subset \Omega$ of $x$ such that $\varphi(x) \in c(j-\Delta)$. The distance $\rho$ between the point $\varphi(x)$ and the closed set $\varphi(J-J)$ is finite and positive. Taking a point $x^{\prime} \in$ $A\left(x, \rho^{\prime} 3\right) \cap S$ and a rational number $\rho^{\prime}: \rho^{\prime} 3<\rho^{\prime}<\rho / 2$, we can see that $x \in 1\left(x^{\prime}, \rho^{\prime}\right)$ $\subset \Omega$ and $.1\left(x^{\prime}, \rho^{\prime}\right) \in \mathrm{r}$. Hence any open set in $E$ is expressed as a union of open sets in $\Xi$. This implies that $\Xi$ is a countable base of open sets in $E$.

2) First we shall remark that there exists an arbitrarily small neighborhood $\Delta$ of $a$, such that the restriction $\varphi_{s}$ of $c$ to $\Delta$ is a proper mapping of $\Delta$ onto

10) From this it follows that our definition of a Riemann domain is equivalent to that of "Riemannsches Gebiet" in H. Grauert [7]. 
some neighborhcod $H$ of $(b)=\varphi(a)$ in $C^{n}$. In fact, since $\varphi$ is non-degenerate at $a$, we can take a relatively compact open neighborhood $\Delta^{\prime}$ of $a$ so that the image of $\bar{\Delta}^{\prime}-\Delta^{\prime}$ does not contain $(b)$. The set $\varphi\left(\bar{\Delta}^{\prime}-\Delta^{\prime}\right)$ is closed subset of $C^{n}$ and, hence, there exists a neighborhood $H$ of $(b)$ containing no point of $c\left(\vec{\Delta}^{\prime}-\Delta^{\prime}\right)$. Then, as is easily seen, $\Delta=\varphi^{-1}(H) \cap \Delta^{\prime}$ satisfies the desired property. Moreover, we may suppose that $\Delta$ is connected and does not contain any point of $\varphi^{-1}(c(a))$ except $a$.

The set $A^{\prime}=\Delta-E_{0}$ is an analytic subset of $\Delta$ and the image $A=\varphi_{\Delta}\left(A^{\prime}\right)$ by the proper mapping $\varphi_{\Delta}$ is an $(n-1)$-dimensional analytic subset of $H$. The mapping $\varphi_{\Delta}$ induces locally a holomorphic isomorphism of $\Delta-\varphi_{\Delta}^{-1}(A)$ onto $H-A$. As $\varphi_{\Delta}$ is proper, the number of points of the set $\varphi_{\Delta}^{-1}((z))$ for any point $(z) \in H-A$ is finite and constant, say $p$. By the definition of an analytic space, a sufficiently small neighborhood $\Delta$ of a can be considered as a normal analytic subset of an open set in some $C^{s}(s$ च $n)$. Accordingly we can construct a holomorphic function $f$ on $\Delta$ vanishing at a and such that, for any pair of point $x, y$ in $\Delta-\varphi_{\Delta}^{-1}(A)\left(x \neq y, \varphi_{\Delta}(x)=\varphi_{\Delta}(y)\right)$, the germs of $f$ at $x$ and $y$ are different (considered as the germs at $\left.\varphi_{\Delta}(x)=\varphi_{\Delta}(y) \in H-A\right){ }^{11)}$ For a point $(z) \in H-A$, the function $f$ determines $p$ functions $f_{j}(j=1,2, \ldots, p)$ on the connected components of $\varphi_{\Delta}^{-1}(N)$ where $N$ is a sufficiently small neighborhood of $(z)$ in $H-A$. Their elementary symmetric functions are holomorphic on $N$, and they are prolongable to one-valued holomorphic functions $a_{i}((z))(i$ $=1,2, \ldots p)$ on $H-A$. As $A$ is an $(n-1)$-dimensional analytic subset of $H$ and $a_{i}((z))$ are locally bounded, $a_{i}((z))$ are also holomorphic on $A$. Hence $f$ is a zero of the distinguished pseudo-polynomial at the center $((b), 0)$ with coefficients holomorphic on $H$ :

$$
P((z), w)=w^{p}+a_{1}((z)) w^{p-1}+\ldots+a_{p}((z)) .
$$

For every connected neighborhood $\Delta^{\prime}$ of $a$ in $E, \Delta^{\prime}-\varphi^{-1}(A)$ is connected, so $P((z), w)$ must be irreducible at $((b), 0)$.

Let $(\tilde{V}, \pi)$ be the Riemann domain of $P((z), w)=0$ and use the same notations as in $\$ 1,2$. The mapping $\varphi_{\Delta} \times f: \Delta \rightarrow H \times C\left(\left(\varphi_{\Delta} \times f\right)(x)=\right.$ $\left.\left(c_{1}(x), f(x)\right), x \in \Delta\right)$ is a proper and holomorphic mapping of $\Delta$ onto $V$. Thus we have the following commutative diagram:

11. More precisely, see Lemma in $\$ 3,2$. 


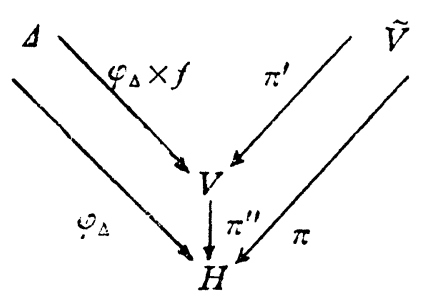

The discriminant set $D(\subset H)$ of $P((z), w)$ clearly contains $A$ and, $\varphi_{\Delta}^{-1}(D)$ and $\pi^{-1}(D)$ are $(n-1)$-dimensional analytic subsets of $\Delta$ and $\tilde{V}$ respectively. Since the restrictions of $\varphi_{\perp} \times f$ to $\Delta-\varphi^{-\frac{1}{\Delta}}(D)$ and of $\pi^{\prime}$ to $\tilde{V}-\pi^{-1}(D)$ are holomorphic isomorphisms into $V$, we obtain the holomorphic isomorphism $\theta_{0}$ of $\Delta-\varphi_{\lrcorner}^{-1}(D)$ onto $\tilde{V}-\pi^{-1}(D)$. Since $\varphi_{\lrcorner} \times f$ (resp. $\left.\pi^{\prime}\right)$ is proper and, for each connected neighborhood $A\left(\right.$ resp..$\left.^{\prime}\right)$ of any point $x \in \varphi_{1}^{-1}(D)$ (resp. $x^{\prime} \in \pi^{-1}(D)$ ) in $\Delta($ resp. $\tilde{V}), 1-\varphi_{\Delta}^{-1}(D)$ (resp. $.1^{\prime}-\pi^{-1}(D)$ ) is also connected, we can extend $\theta_{0}$ to a homeomorphism $\theta$ of $\lrcorner$ onto $\widetilde{V}$ such that $\varphi_{\lrcorner}=\pi^{\circ} \theta$. From a well known theorem of removable singularities, it follows that $\theta$ is a holomorphic isomorphism. That is, the continuous mapping $\theta$ (resp. $\left.\theta^{-1}\right)$ on $\Delta($ resp. $\tilde{V})$ is holomorphic not only on $\Delta-c_{\Delta}^{-1}(D)$ (resp. $\widetilde{V}-\pi^{-1}(D)$ ) but also at every uniformisable point of $\Delta$ (resp. $\tilde{V})$. q.e.d.

3. In the following considerations the notion of a boundary point of a Riemann domain plays an impotant role.

Definition 2. A boundary point ${ }^{12)}$ of a Riemann domain $(E, \varphi)$ is a filter $^{13)} r=\{A\}$ in $E$ which consists of open connected subsets of $E$ and satisfies the following conditions.

1) The image filter $\{\varphi(d), d \in r\}$ of $r$ converges to a point $(z) \in C^{n}$.

2) For every open neighborhood $N$ of the point $(z) \in C^{n}$, the filter $r$ containts a connected component of $\varphi^{-1}(N)$ and consists of only such sets.

3) The filter $r$ has no accumulating point in $E^{14)}$

The set of all boundary points of $(E, \varphi)$ is called the (accessible) boundary of $(E, \varphi)$ and denoted by $\partial E$. Let us put $\check{E}=E \cup \partial E$. The mapping $\varphi$ is

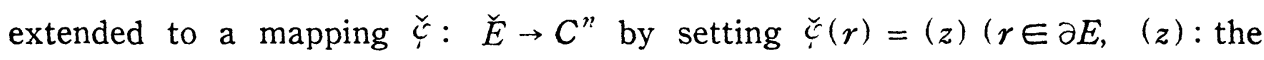

12) This definition is due to H. Grauert and R. Remmert [8] and is equivalent to that of "Randpunkt" in H. Behnke and P. Thullen [2] Chapt. I, \$ 3.

13) Here we call "a base of filter" in usual sense "a filter" simply.

14) This means that any directed set determined by $r$ does not converge in $E$. 
limiting point of the filter $\{\varphi(.1), .1 \in r\})$. Of course, the boundary $\partial E$ of $(E, \varphi)$ depends upon $\varphi$. Even if $(E, \varphi)$ is univalent (i.e. $E$ is an open set in $C^{n}$ and $\varphi$ is the canonical injection of $E$ into $\left.C^{n}\right), \breve{E}$, in general, does not coincide with the closure $\bar{E}$ of $E$ in $C^{n}$.

We shall introduce a topology on $\check{E}$ which induces on $E$ the same topology as the original one of $E$, by defining neighborhoods of every boundary point $r \in \partial E$. Let $A$ be an open set in $r$ and let $\breve{1}$ be the union of $A$ and all the boundary points whose filters contain at least one set contained in $A$. We define the system of such sets $1(1 \in r)$ as the base of neighborhoods of $r$.

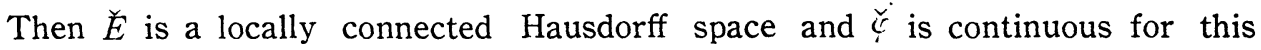
topology. Moreover, we have

TheOREM 2. For a Riemann domain $(E, \varphi)$, the space $\check{E}=E \cup \partial E$ satisfies the second countability axiom.

Proof. Since $E$ satisfies the second countability axiom by Theorem 1, the number of connected components of $\varphi^{-1}(H((z), \rho))$ is countable for every hypersphere $H((z), \rho)$ with radius $\rho$ around $(z) \in C^{n}$. The set $\check{\zeta}^{-1}(H((z), \rho))$ has also countable connected components. In fact, the relation $\check{\varphi}^{-1}(H((z), \rho))$ $\cap E=\varphi^{-1}(H((z), \rho))$ holds and the intersection of a connected component of $\check{c}^{-1}(H((z), \rho))$ and $E$ is connected.

Now, consider the countable system $S$ of open sets in $\check{E}$ consisting of all connected components of $\check{\varphi}^{-1}(H((z), \rho))$ when $(z)$ and $\rho$ run over the set of all points with rational coordinates and the set of all positive rational numbers respectively. Then $\Xi$ is a base of open sets in $\breve{E}$, that is, any open set $\Omega$ in $\breve{E}$ can be expressed as a union of sets in $\Xi$. To prove this it suffices to show that, for every boundary point $r \in \Omega$, there exists a set $\Delta$ containing $r$ in $\Xi$ such that $\Delta \subset \Omega$. As $\Omega$ is open, there exists a neighborhood of $r$ contained in $\Omega$. By the definition of the topology on $E$, this neighborhood is of type $\check{1}$, where $.1 \in r$ i.e., $l$ is a connected component of the inverse image by $\varphi$ of an open neighborhood $N$ of $\check{\zeta}(r) \in C^{n}$. We can take a hypersphere $H((z), \rho)$ with the radius $\rho$ around a rational point $(z) \in C^{n}$ such that $\breve{\zeta}(r) \in H((z), \rho) \subset N$. Let $\Delta$ be the connected component of $\check{\zeta}^{-1}(H((z), \rho))$ containing $r$. Then it is easy to verify that $r \in \Delta \subset \check{1} \subset \Omega$ and $\Delta \in \Xi$. q.e.d.

From this theorem the subspace $\partial E$ of $\check{E}$ satisfies the second countability. 
axiom for the induced topology. Hence $\partial E$ is a separable topological space. Thus we have.

Corollary. There exists a countable dense set in $\partial E$.

\section{$\S 3$. Holomorphic prolongations and domains of holomorphy.}

1. Let $E, E^{\prime}$ be analytic spaces and let $D(E), \mathscr{D}\left(E^{\prime}\right)$ be the rings of all holomorphic functions on $E, E^{\prime}$ respectively.

For a given holomorphic mapping $\lambda: E \rightarrow E^{\prime}$, we define a mapping $\lambda^{\star}$ : $\mathfrak{D}\left(E^{\prime}\right) \rightarrow \mathfrak{D}(E)$ by setting $\lambda^{*}\left(f^{\prime}\right)=f^{\prime} \circ \lambda\left(f^{\prime} \in \mathfrak{D}\left(E^{\prime}\right)\right)$. If $\lambda$ is an open mapping, $\lambda^{*}$ is one to one by the theorem of identity.

Definition 3. Let $\lambda: E \rightarrow E^{\prime}$ be a given holomorphic mapping. If a function $f \in \mathfrak{S}(E)$ belongs to the image of $D\left(E^{\prime}\right)$ by $\lambda^{*}$ (i.e. there exists a function $f^{\prime} \in \mathbb{D}\left(E^{\prime}\right)$ such that $\left.f=f^{\prime} \circ \lambda\right)$. $f$ will be called holomorphically prolongable to $E^{\prime}$ with respect to $\lambda\left(f^{\prime}\right.$ will be called a holomorphic prolongation of $f$ to $E^{\prime}$ with respect to $\lambda$ ).

Let $(E, C)$ and $\left(E^{\prime}, \varphi^{\prime}\right)$ be two Riemann domains spred over $C^{n}$. If there exists a holomorphic mapping $\lambda: E \rightarrow E^{\prime}$ such that $\varphi=\varphi^{\prime} \circ \lambda$, we say for convenience sake that $(E, \varphi)$ is contained in $\left(E^{\prime}, \varphi^{\prime}\right)$. Then, as $\lambda: E \rightarrow E^{\prime}$ is nondegenerate at every point of $E, \lambda$ is an open mapping. ${ }^{15)}$ Hence the holomorphic prolongation of a holomorphic function on $E$ to $E^{\prime}$ is uniquely determined if it exists.

2. Let $(E, \varphi)$ be a Riemann domain and let $f$ be a holomorphic function on it given once for all. In the following we shall consider holomorphic prolongations of $f$ to those Riemann domains which contain $(E, \varphi)$. In the first place we prove the following theorem which is a generalization of classical one for an unramified Riemann domain. ${ }^{16)}$

TheOrem 3. Let a Riemann domain $(E, \varphi)$ and a holomorphic function $f$ on it be given. Then there exists a maximal one in the family of all Riemann domains containing $(E, C)$ to which $f$ is holomorphically prolongable. More

15) Cf. R. Remmert [11] Satz 28.

16. Cf. H. Cartan [4] VII. As for this generalization, R. Iwahashi has considered in [10] more generally for a domain spred on an analytic space using a space of algebroidal jets, 
precisely we can canonically construct a Riemann domain $(\widetilde{E}, \widetilde{c})$ with the following properties; 1) there exists a holomorphic mapping $\psi: E \rightarrow \widetilde{E}$ and a holomorphic function $\widetilde{f}$ on $\widetilde{E}$ sush that $\varphi=\widetilde{\varphi}^{\circ} \psi$ and $\left.f=\widetilde{\zeta} \circ \psi, 2\right)$ for every Riemann domain $\left(E^{\prime}, \varphi^{\prime}\right)$ with a holomorphis mapping $\lambda: E \rightarrow E^{\prime}$ and a holomorphic function $f^{\prime}$ on $E^{\prime}$, satisfying relations $\varphi=\varphi^{\prime} \circ \lambda, f=f^{\prime} \circ \lambda$, there exists a holomorphic mapping $\psi^{\prime}: E^{\prime} \rightarrow \widetilde{E}$ so that $\varphi^{\prime}=\widetilde{\varphi} \circ \psi^{\prime}, f^{\prime}=\widetilde{f} \circ \psi^{\prime}$, and $\psi=\psi^{\prime} \circ \lambda$.

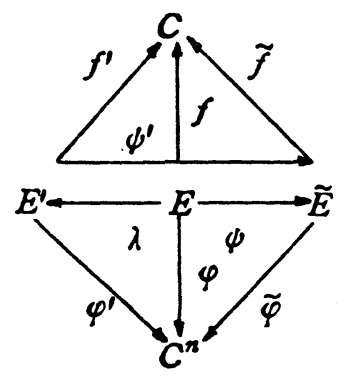

Proof. To construct $(\widetilde{E}, \widetilde{\varphi})$, we begin with a consideration on the space $X$ consisting of all germs of analytic subsets of algebroidal functions. For a point $(b) \in C^{n}$ and a constant $c$, we consider an irreducible distinguished pseudopolynomial at the center $((b), c)$ with coefficients holomorphic at $(b)$ :

$$
P((z), w)=(w-c)^{p}+a_{1}((z))(w-c)^{p-1}+\ldots+a_{p}((z)) .
$$

Let $V$ be the analytic subset of $H \times C$ defined by $P((z), w)=0, H$ being a neighborbhood of $(b) \in C^{\prime \prime}$, and let $V_{\langle(b), c)}$ be the germ of $V$ at $((b), c)$.

Now we shall define the topological space $X$ formed by all such germs $V_{((b), c)}$ at $((b), c)$ where $(b)$ and $c$ run over $C^{n}$ and $C$ respectively. The topology on $X$ is as follows. Suppose that a point $V_{((b), c)} \in X$ is defined as above and $(\tilde{V}, \pi)$ is the Riemann domain of the algebroidal function $P((z), w)$ $=0$ on $H$. Then, for each neighborhood $N$ of $(b)$ contained in $H, \pi^{-1}(N)$ may be considered as a subset of $X$. The system of such subset defines the base of neighborhoods of $V_{(\langle b), c)}$ for the topology on $X$. So $\tilde{V}$ may be considered as an open subset of $X$ and the injection of $\hat{V}$ into $X$ is a homeomorphism. As is easily seen, $X$ is a Hausdorff space.

Let $\Phi: X \rightarrow C^{n}$ be a continuous mapping defined by $\Phi\left(V_{((b), c)}\right)=(b)^{\circ}$. By the mapping $\emptyset$ we can introduce on the Hausdorff space $X$ a structure of an analytic space. In fact, for each point $V_{((b), c)} \in X$, there exists the Riemann domain $(\tilde{V}, \pi)$ so that $\widetilde{V}$ coincides with an open neighborhood of $V_{((b), c)}$ 
including the topological structure, and $\pi$ coincides with $\Phi$ on $\tilde{V}$. Since the analytic structure of $\bar{V}$ is determined uniquely by the topological structure of $\widetilde{V}$ and the mapping $\pi(\$ 1,2)$, we see by transfering the analytic structure of $\dot{V}$ to $X$ that $X$ becomes an analytic space. As $\mathscr{D}$ is non-degenerate at every point of $X$, the pair $(X, \emptyset)$ is a (non-connected) Riemann domain.

Next we define a holomorphic function $F$ on $X$ by associating the value $c$

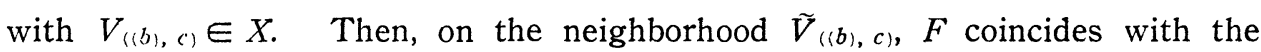
holomorphic function associated with the algebroidal function $P((z), w)=0$ $(\S 1,2)$.

Furthermore, we shall define a holomorphic mapping $\Psi$ of $E$ into $X$ such that relations $\varphi=\emptyset \circ \Psi$ and $f=F \circ \Psi$ hold. For this purpose, it is to be noticed that on a suitable neighborhood of each point $a \in E$, the given function $f$ satisfies a polynomial equation $P((z), w)=0$ (i.e. $P(\varphi(x), f(x))=0$ for every point $x$ in this neighborhood), where $P((z), w)$ is an irreductible distinguished pseudo-polynomial of the center $(\varphi(a), f(a))$ with coefficients holomorphic at $(b)=c(a)$. In fact, taking a suitable connected neighborhood $\Delta$ of $a$, we see by Theorem 1 that $\left(\Delta, \varphi_{s}\right)$ is isomorphic to the Riemann domain of an algebroidal function on a neighborhood $H$ of $(b)$. Hence, for a suitable $(n-1)$. dimensional analytic subset $A$ of $H$, the number of sheets of $\Delta-\varphi^{-1}(A)$ over $H-A$ is finite and constant. As in the proof of Theorem 1,2$)$, we see that $f$ satisfies $Q((z), w)=0$ on $\Delta$, where $Q((z), w)$ is a distinguished pseudopolynomial at the center $((b), f(a))$ with coefficients holomorphic on $H$. Then $Q((z), w)$ must be some power of an irreducible pseudo-polynomial $P((z, w)$ with coefficients holomorphic at $(b)$, since for any connected sufficiently small neighborhood $\Delta^{\prime}$ of $a, \Delta^{\prime}-\varphi^{-1}(A)$ is also connected. Let $\left.V_{(b)}, f(a)\right)$ be the germ at $((b), f(a))$ of the analytic subset defined by $P((z), w)=0$. By definition, the mapping $\Psi: E \rightarrow X$, associates $a \in E$ with the above germ $V_{((b), f(a)) .}$ It is easy to verify that $\Psi$ is the required mapping.

Let $\widetilde{E}$ be the connected component of $X$ containing the (connected) image of $E$ by $\Psi$, let $\psi$ be the holomorphic mapping of $E$ into $\widetilde{E}$ determined by $\Psi$, and let $\widetilde{c}, \widetilde{f}$ be the restrictions to $E$ of $\mathscr{D}, F$ respectively. Then the Riemann domain $(\widetilde{E}, \tilde{c})$ and mapping $\psi, \widetilde{f}$ satisfy the property 1$)$ in the theorem. As for 2), we define similarly the holomorphic mapping $\Psi^{\prime}: E^{\prime} \rightarrow X$, considering $\left(E^{\prime}, \varphi^{\prime}\right)$ insted of $(E, \zeta)$. From the assumption on $\left(E^{\prime}, \varphi^{\prime}\right)$, it follows that 
$\Psi(E) \subset \Psi^{\prime \prime}\left(E^{\prime}\right) \subset \widetilde{E} \subset X$. Thus we obtain the mapping $\psi^{\prime}: E^{\prime} \rightarrow \widetilde{E}$ which possesses the property 2 ). q.e.d.

3. The Riemann domain $(\widetilde{E}, \widetilde{Y})$ constructed as above is called the Riemann domain of the maximal holomorphic prolongation of $f$ or the existence domain of $f$, and $\tilde{f}$ is called the maximal holomorphic prolongation of $f$.

Suppose that two Riemann domains $(E, \varphi)$ and $\left(E^{\prime}, \varphi^{\prime}\right)$ are isomorphic by $\lambda: E^{\prime} \rightarrow E$. Then the existence domain of a holomorphic function $f$ on $E$ is isomorphic to the existence domain of the holomorphic function $f^{\prime}=f \circ \lambda$ on $E^{\prime}$. Hence we can define the notion of a Riemann domain of holomorphy as follows :

Definition 4. Let $(E, \varphi)$ be a Riemann domain. If there exists a holomorphic function $f$ on $E$ such that the existence domain of $f$ is canonically isomorphic to $(E, \varphi)$, we say $(E, \varphi)$ to íe a (Riemann) domain of holomorphy."i)

\section{§4. A sufficient condition for a Riemann domain to be a domain of holomorphy.}

1. Let $E$ be an analytic space and $\tilde{j}$ be a family of holomorphic functions on $E$. For a compact set $K$ in $E$, we put

$$
\hat{K} \preccurlyeq=\left\{x \in E /|f(x)| \leqq \sup _{y \in K}|f(y)| \text { for all } f \in \tilde{\mho}\right\} .
$$

Then $\hat{K}_{\Re}$ is a closed subset of $E$. When $\tilde{F}$ consists of all holomorphic functions on $E$, we denote $\hat{K}_{\Im}$ by $\hat{K}$ simply, and call it the envelope of holomorphy of $K$.

Definition 5. We say that a Riemann domain $(E, \varphi)$ satisfies the condition (T) if 1) for every pair of points $x, y \in E(x \neq y \varphi(x)=\varphi(y)=(z))$ there exists a holomorthic function $f$ on $E$ such that two germs of $f$ at $x$ and $y$ are different (considered as the germs of algebroidal functions at $(z)$ ), and 2) for every compact set $K$ in $E$ and every boundary point $r$ of $(E, \varphi)$, the envelope of holomorphy $\hat{K}$ contains no set in the filter defining $r$.

An analytic space $E$ is said to be holomorphisally convex if, for any compact set $K$ in $E, \hat{K}$ is again compact. A Riemann domain is holomorphically convex if the analytic space $E$ is holomorphically convex.

17) In the unramified case it is known that if one of two Riemann domains $(E, \phi)$, $\left(E^{\prime}, \phi^{\prime}\right)$ with holomorphic isomorphic $E, E^{\prime}$, is a domain of holomorphy, so is the other. But this does not hold in general. Cf. H. Grauert and R. Remmert [8]. 
The condition $(\Gamma)$ is strictly weaker than the holomorphic convexity, in fact, it is known that if a Riemann domain $(E, \varphi)$ is holomorphically convex, then, for any pair of distinct points $x, y \in E$, there exists a holomorphic function $f$ on $E$ such that $f(x) \neq f(y){ }^{1 \text { (s) }} \quad$ Thus, in particular, a holomorphically convex Riemann domain $(E, \varphi)$ satisfies the condition 1$)$ in Definition 5 , while it satisfies clearly the condition 2). Furthermore we can verify that the example in [8] of a Riemann domain of holomorphy which is not holomorphically convex, satisfies the condition $(\Gamma)$. It will be seen later on that in the unramified case the condition $(\Gamma)$ is equivalent to the holomorphic convexity.

2. Now we shall prove the following

THEOREM 4. The condition ( $\Gamma$ ) (in particular, the holomorphic convexity) is a sufficient condition for a Riemann domain to be a domain of holomorphy.

Proof. Let $(E, \varphi)$ be a Riemann domain. By Corollary of Theorem 2, there exists a countable dense set $S=\left\{\boldsymbol{r}_{1}, \boldsymbol{r}_{2}, \ldots\right\}$ in the boundary $\partial E$ of $(E, \zeta)$. Each boundary point $r_{i} \in S$ (more precisely the filter defining $r_{i}$ ) contains an uniquely determined connected component $\lambda_{j}^{\left(r^{(}\right)}$of the inverse image by $c$ of a

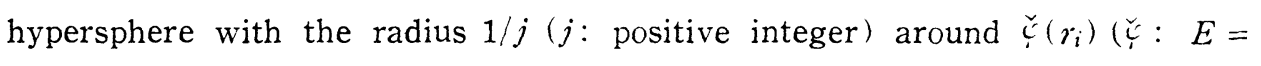
$E \cup \partial E \rightarrow C^{n}$, see $\left.\S 2,3\right)$. Thus we obtain the sequence of open sets in $r_{i}$ : $\left\{A_{j}^{\left(r_{i}\right)}\right\}_{j=1,3} \ldots$. Now, consider the following sequence :

$$
A_{1}^{\left(r_{1}\right)}, A_{1}^{\left(r_{2}\right)}, A_{2}^{\left(r_{1}\right)}, A_{1}^{\left(r_{3}\right)}, A_{2}^{\left(r_{2}\right)}, A_{3}^{\left(r_{1}\right)}, A_{1}^{\left(r_{1}\right)}, A_{2}^{\left(r_{3}\right)}, A_{3}^{\left(r_{2}\right)}, A_{4}^{\left(r_{1}\right)}, A_{1}^{\left(\gamma^{3}\right)}, A_{2}^{\left(r_{3}\right)}, \ldots
$$

and denote by $1_{k}$ the $k$-th term of this sequence.

On the other hand, as $E$ satisfies the second countability axiom (Theorem 1,1 , there exists an increasing sequence $\left\{K_{k}\right\}_{k=1,2} \ldots$ of compact sets in $E$ such that $E=\cup K_{k}$. Assume that the Riemann domain $(E, \varphi)$ satisfies the condition $(\Gamma)$. Then for each $k$ there exists a point $x_{k}$ in $l_{k}$ such that $x_{k} \notin K_{k}$. Hence the inequality $\sup _{K_{k}}\left|f_{k}\right|<\left|f_{k}\left(x_{k}\right)\right|$ holds for some holomorphic function $f_{k}$ on $E$. We may suppose $f_{k}\left(x_{k}\right)=1$. Denoting, if necessary, a suitable power of $f_{k}$ again by $f_{k}$, we have

$$
\sup _{K_{k}}\left|f_{k}\right|<2^{-k} \text { for each } k \text {. }
$$

Then the infinite product $\Pi_{k}\left(1-f_{k}\right)^{k}$ converges uniformly on every $K_{k}$ and

18) Cf. H. Grauert [7] 
therefore, it defines a holomorphic function $f$ on $E$.

The point $x_{k} \in A_{k}$ is a zero-point of $f$ of order $\triangleq k$. Consequently, $f$ has a zero-point of arbitrarily high order in each $A_{j}^{\left(r_{i}\right)}\left(r_{i} \in S\right)$. Let $r$ be an arbitrary boundary point. Since $S$ is dense in $\partial E$, any neighborhood $\check{L}(\Lambda \in r)$ of $r$ in $\check{E}$ contains a boundary point $r_{i} \in S$. From the definition of the topology on $\breve{E}$ it follows that $\Lambda \in r$ contains an open set $\Lambda_{j}^{\left(r_{i}\right)}$ in $r_{i}$. Therefore we can conclude that the holomorphic function $f$ has a zero-point of arbitrarily high order in every open set $A$ in the filter defining each boundary point $r$ of $(E, \varphi)$.

Now we use the following lemma which will be proved afterwards.

Lemma. Suppose that a family $\mathfrak{F}$ of holomorphic functions on a Riemann domain $(E, \varphi)$ satisfies the condition that, for any pair of point $x, y \in E(x \neq y$, $\varphi(x)=\varphi(y))$, there exists a function $f$ in $\tilde{F}$ such that the germs of $f$ at $x$ and $y$ are different. Then there exists a series $g=\sum_{k} \lambda_{k} g_{k}\left(\lambda_{k}\right.$ : complex numbers, $g_{k} \in \widetilde{F}_{k}$ ) convergent uniformly on every compact set in $E$ such that $g$ has different germs at $x$ and $y$ for any pair $x, y$ such that $x \neq y, \varphi(x)=\varphi(y)$.

Let us denote by $\mathfrak{f}$ the family consisting of all the holomorphic functions on $E$ which are divisible by the above $f$. Then $\mathfrak{f}$ satisfies clearly the assumption in Lemma. Hence there exists a holomorphic function $g=$ $\sum \lambda_{k} g_{k}\left(g_{k} \in \mathscr{F}\right)$ on $E$ such that :

(1) the germs of $g$ at $x$ and $y$ are different for any pair of points $x, y$ $\in E(x \neq y, \varphi(x)=\varphi(y))$.

(2) the function $g$ has a zero-point of arbitrarily high order in every open set in the filter defining each boundary point.

Let $(\widetilde{E}, \widetilde{\zeta})$ be the existence domain of $g$ and let $\psi$ be the canonical mapping of $E$ into $\widetilde{E}$. To complete the proof it suffices to show that $\psi$ is an isomorphism between $(E, \varphi)$ aud $(\widetilde{E}, \widetilde{\varphi})$. From the construction of $\psi(\S 3,2)$ and the property (1), $\psi$ is clearly an analytic isomorphism into $\widetilde{E}$. The mapping $\psi: E \rightarrow \widetilde{E}$ satisfying the relation $\varphi=\widetilde{\varphi} \circ \psi$, can be extended to the mapping $\tilde{\psi}: E \cup \partial E \rightarrow$ $\widetilde{E} \cup \partial \widetilde{E}$ in natural way. Then it can be verified that $\tilde{\psi}(\partial E) \subset \partial \widetilde{E}$. In fact, assume that, for a boundary point $r \in \partial E, \breve{\psi}(r)$ were in $\widetilde{E}$. then $g$ would define a germ of algebroidal function at $\breve{\psi}(r)=\widetilde{\varphi}(\breve{\psi}(r)) \in C^{n}$. Hence, $H$ being a sufficiently small neighborhood of $\check{\varphi}(r)$ and $A$ being the connected component of $\varphi^{-1}(H)$ belonging to the filter $r$, the orders of zero-points of $g$ in 1 would be 
bounded. This contradicts the property (2). The reiation $\breve{\psi}(\partial E) \subset \partial \widetilde{E}$ implies that $\psi$ is an isomorphism of $E$ onto $\widetilde{E}$, for, if there exists a point $x$ in $\widetilde{E}-\psi(E)$, a path joining $x$ to a point in $\psi(E)$ would define a boundary point $r$ of $(E, \varphi)$ in the sense of Definition 2 such that $\breve{\psi}(\boldsymbol{r}) \notin \partial \widetilde{E}$. Thus the theorem has been proved.

Proof of Lemma. Let $\left(E_{0}, \varphi_{0}\right)$ be the unramified Riemann domain associated with $(E, \varphi)$. Then $E_{0}$ can be covered by a sequence of open sets $\Delta_{i}$ $(i=1,2, \ldots)$ which are homeomorphic to some hyperspheres in $C^{n}$ by $\varphi_{0}$. We may assume that the system $\left\{\Delta_{i}\right\}_{i=1,2}, \ldots$ is a fundamental system of open sets in $E_{0}$, since $E_{0}$ satisfied second countability axiom. It is easily seen from the assumption on $\mathfrak{F}$ that, for any couple $\left(\Delta_{i}, \Delta_{j}\right)\left(\Delta_{i} \cap \Delta_{j}=\phi, \varphi_{0}\left(\Delta_{i}\right) \cap \varphi_{0}\left(\Delta_{j}\right) \div \phi\right)$, we can choose a couple of points $x_{i j} \in \Delta_{i}, x_{j i} \in \Delta_{j}$ and a function $g_{i j} \in \mathfrak{\jmath}$ such that $\varphi_{0}\left(x_{i j}\right)=\varphi_{0}\left(x_{j i}\right)$ and $g_{i j}\left(x_{i j}\right)=g_{i j}\left(x_{j i}\right)$. Arrange the functions $g_{i j}$ in a simple sequence: $g_{1}, g_{2}, \ldots$ On the other hand, $E$ is a union of an increasing sequence of compact sets $K_{k}(k=1,2, \ldots)$ in $E$. If the constants $\lambda_{k}$ are chosen so that $\sup _{K_{k}}\left|\lambda_{k} g_{k}\right| \leqq 2^{-k}$ for each $k$, the series $\sum \lambda_{k} g_{k}$ converges absolutely and uniformly on every compact set in $E$. Moreover we can take $\lambda_{k}$ to satisfy the supplementary condition that $\sum \lambda_{k}\left(g_{k}\left(x_{i j}\right)-g_{k}\left(x_{j i}\right)\right) \neq 0$ for any couple $(i, j)\left(\Delta_{i} \cap \Delta_{j}=\phi, \varphi_{0}\left(\Delta_{i}\right) \cap \varphi_{0}\left(\Delta_{j}\right) \neq \phi\right)$. In fact, let us consider the linear inequalities with respect to $\xi_{k}: \sum \xi_{k}\left(g_{k}\left(X_{i j}\right)-g_{k}\left(x_{j i}\right)\right) \geq 0$. Since at least one of their coefficients is not equal to zero, the countable system of inequalities holds for $\hat{\xi}_{k}=\lambda_{k}(k=1,2, \ldots)$ with arbitrarily small absolute values. Thus the holomorphic function $g=\sum \lambda_{k} g_{k}$ on $E$ has distinct values at $x_{i j}$ and $x_{j i}$. For any pair of points $x, y \in E_{0}\left(x \neq y \quad \varphi_{0}(x)=\varphi_{0}(y)\right)$, there exists a couple $\left(\Delta_{i}, \Delta_{j}\right)$ such that $x \in \Delta_{i}, y \in \Delta_{j}, \Delta_{i} \cap \Delta_{j}=\phi, \varphi_{0}\left(\Delta_{i}\right) \cap \varphi_{0}\left(\Delta_{j}\right) \neq \phi$. Then the germs $g_{\mathfrak{r}}, g_{y}$ of $g$ at $x$ and $y$ are differet since otherwise $g$ would define the same germs at $x_{i j} \in \Delta_{1}$ and $x_{j i} \in \Delta_{j}$ by analytic prolongations of $g_{x}$ and $g_{i}$. As $E_{0}$ is dense in $E$, the series $g=\sum \lambda_{k} g_{k}$ satisfies the requirement of our lemma.

Remark. By a theorem of $\mathrm{K}$. Oka ${ }^{19)}$ an unramified Riemann domain of holomorphy is holomorphically convex, hence satisfies $(\Gamma)$. Therefore in the unramified case the holomorphic convexity and the condition $(\Gamma)$ are equivalent, and they are both sufficient and necessary conditions for a Riemann domain to

19) Cf. K. Oka [6]. 
be a domain of holomorphy.

3. As an application of Theorem 4, we shall remark on the convergence problem of domains of holomorphy. An ordered pair of Riemann domain $(E, \varphi),\left(E^{\prime}, \varphi^{\prime}\right)$ is said to be a Runge pair if 1$) E$ is an open subset of $E^{\prime}$ and $\varphi^{\prime}$ coincides with $\varphi$ on $E, 2$ ) any holomorphic function on $E$ is approximated uniformly on every compact set in $E$ by holomorphic functions on $E^{\prime}$.

It is known that for a sequence of holomorphically convex unramified Riemann domains such that each ordered pair of adjacent terms is a Runge pair, the limiting (unramified) Riemann domain is holomorphically convex. ${ }^{20)}$

As a generalization of this result we prove the following.

Theorem 5. For a sequence $\left\{\left(E_{\nu}, \varphi_{\nu}\right)\right\}_{\nu=1,2, \ldots}$ of Riemann domains satisfying $(\Gamma)$ such that each ordered pair $\left(E_{\nu}, \varphi_{\nu}\right),\left(E_{\nu+1}, \varphi_{\nu+1}\right)$ is a Runge pair, the limiting Riemann domain $(E, \varphi)$ satisfies $\left(I^{\prime}\right)$, and hence, is a domain of holomorphy.

Proof. First, we shall show that, for each $\nu_{0}$ the pair $\left(E_{\nu_{0}}, \varphi_{\nu_{0}}\right),(E, \varphi)$ is a Runge pair. Let $f_{v_{0}}$ be a holomorphic function on $E_{v_{0}}$ and let $K_{v_{0}}$ be a compact set in $E_{\nu_{0}}$. Take a compact set $K_{\nu}$ in $E_{\nu}$ for each $\nu \geqslant \nu_{0}$ such that $K_{\nu}$ $C K_{\nu+1}\left(\nu \geqslant \nu_{0}\right)$ and $E=\cup_{\imath \geqq \nu} K_{\nu}$. As the pair $\left(E_{\nu,}, \varphi_{\nu}\right),\left(E_{\nu+1}, \varphi_{\nu+1}\right)$ is a Runge pair there exists a holomorphic function $f_{\nu}$ on $E_{\nu}$ and $\left|f_{\nu}-f_{\nu+1}\right| \leqq \varepsilon_{\nu}$ on $K_{\nu}$ for an arbitrary positive number $\varepsilon_{\nu}$ for each $\nu \supseteq \nu_{1}$. Consequently the sequence $\left\{f_{v}\right\}_{\left\llcorner\geqq v_{0}\right.}$ converges uniformly every compact set of $E$ to a holomorphic function $f$ on $E$ and $\left|f-f_{\nu_{0}}\right| \leqq \sum \sum_{\nu_{0}} \varepsilon_{\nu}$ holds on $K_{\nu_{0}}$, where $\sum_{\nu_{2 \nu_{0}} \varepsilon_{\nu}}$ can be taken arbitrarily small. This means that $f_{v_{0}}$ is approximated uniformly on $K_{v_{0}}$ by holomorphic functions on $E$. Hence the pair $\left(E_{v_{v}}, \varphi_{v_{0}}\right),(E, \varphi)$ is a Runge pair.

Next let $K$ be a compact set in $E$. Denoting by $\hat{K}$ and $\bar{K}$ the enveloppes of holomorphy of $K$ in the space $E$ and a space $E_{\nu_{0}}$ containing $K$ respectively, we have $\bar{K}=\hat{K} \cap E_{\nu_{0}}$. To prove this it suffices to verify that $\bar{K} \supset \hat{K} \cap E_{\nu_{0}}$, because $\bar{K}$ is obviously contained in $\hat{K} \cap E_{\nu_{0}}$. Suppose that a point $x \in E_{v_{0}}$ does not belong to $\bar{K}$, then there exists a holomorphic function $f_{v_{0}}$ on $E_{\nu_{0}}$ so that $\left|f_{\nu_{0}}(x)\right|>\sup _{K}\left|f_{\nu_{0}}\right| . \quad$ Since the pair $\left(E_{\nu_{0}}, \varphi_{\nu_{0}}\right),(E, \varphi)$ is a Runge pair as shown above, $f_{\nu_{0}}$ be approximated uniformly on the compact set $\{x\} \cup K$ in $E_{v_{0}}$ by

20) Cf. H. Behnke and K. Stein [3]. 
holomorphic functions on $E$. "Hence we can find a holomorphic function $f$ on $E$ such that $|f(x)|>\sup _{\kappa}|f|$. This means that the point $x$ does not belong to $\hat{K}$.

Now let $r$ be a boundary point of $(E, \varphi)$. Let $A$ be a set in $r$ and let $K$ be a compact set in $E$. As $(E, \varphi)$ is the limiting Riemann domain of $\left\{\left(E_{\nu}, \varphi_{\nu}\right)\right\}$, there exists a Riemann domain $\left(E_{\nu_{0}}, \varphi_{\nu_{0}}\right)$ and a boundary point $r^{\prime}$ of it such that $K$ is contained in $E_{v_{0}}$ and $A$ contains some $\Lambda^{\prime}$ in $r^{\prime}$. Since the envelope of holomorphy $\bar{K}$ of $K$ in $E_{\nu_{0}}$ coincides with $E_{\nu_{0}} \cap \hat{K}$ as mentioned above, we have $A-\hat{K} \supset(A-\hat{K}) \cap E_{0} \supset A^{\prime}-\bar{K}$. Since $\left(E_{\nu_{0}}, \phi_{\nu_{0}}\right)$ satisfies $\left(I^{\prime}\right)$ by the assumption, the set $A^{\prime}-\bar{K}$ is non-empty, and so is $A-\bar{K}$. This implies that $(E, \varphi)$ satisfies $(\Gamma)$. q.e.d.

4. The condition $(\Gamma)$ is necessary and sufficient for an unramified Riemann domain to be a domain of holomorphy (Remark in $\$ 4,2$ ). In general ramified cases, we know in Theorem 4 that the condition $(\Gamma)$ is again sufficient for a Riemann domain to be a domain of holomorphy. However it is not yet clarified whether this condition $(\Gamma)$ is also necessary. On the necessity of $(\Gamma)$ we shall make a remark in the following.

Let $(E, \varphi)$ be a Riemann domain and let $\left(E_{0}, \varphi_{0}\right)$ be the unramified Riemann domain associated with $(E, \varphi)$. Denoting by $\mathfrak{D}\left(E_{0}\right)$ the set of all holomorphic functions on $E_{0}$, we call a subset $\Re$ of $\subseteq\left(E_{0}\right)$ a holomorphic class on the unramified Riemann domain $\left(E_{0}, \varphi_{0}\right)$ if $\Re$ is an algebra over the complex number field and is stable by the differentiations with respect to the local coordinates defined by $\varphi_{0}$. We always consider on $D\left(E_{0}\right)$ the uniform convergence topology (on every compact set in $E_{0}$ ). Then we have

Theorem 6. Let $(E, \varphi)$ be a Riemann domain of holomorphy, i.e., the existence domain of a holomorphic function $f$ on $E$. Suppose that there exists a holomorphic class $\mathfrak{A}$ on $\left(E_{0}, \varphi_{0}\right)$ containing the restriction $f_{0}$ of $f$ to $E_{0}$ such that every function in $\mathfrak{R}$ is holomorphically prolongable to $E$ and $\mathfrak{R}$ is closed in $\mathcal{D}\left(E_{0}\right)$. Then $(E, \varphi)$ satisfies the condition $(\Gamma)$. Furthermore $(E, \varphi)$ is unramified.

Proof. First of all, we prove that if every function in $R$ is bounded on a subset $\Sigma$ of $E_{0}$, then there exists a compact set $K_{0}$ in $E_{0}$ such that the set $\hat{K}_{0 \Omega}$ $=\left\{x \in E_{0} /|h(x)| \leqq \sup _{K_{0}}|h|\right.$ for all $\left.h \in \Re\right\}$ contains $\Sigma$. Considering the induced 
topology on $\Re \subset D\left(E_{0}\right), \Re$ is a metrisable and complete topological space, because $\mathscr{R}$ is closed in $\mathscr{O}\left(E_{0}\right)$. Therefore $\mathscr{R}$ is a Baire space. Associate $\sup _{\Sigma}|h|$ with $h \in \Re$. Then we obtain a lower semi-continuous function $\tau$ on $\AA$. Hence, by the Theorem of Baire, $\tau$ is bounded uniformly on some non-empty open set $\mathfrak{U} \subset \mathfrak{A}$. We may choose the set $\mathfrak{U}$ as a neighborhood of the zero function in $\Re$, by translation, if necessary. That is, there exists a compact set $K_{0}$ in $E_{0}$ such that, for any function $h$ in $\Re, \sup _{K_{0}}|h| \leqq 1$ implies $\sup _{\Sigma}|h| \leqq M$, where $M$ is a fixed positive number. As this holds for any power of $h, M$ can not be larger than 1. Thus we see that $\sup _{K_{0}}|h| \leqq 1$ implies $\sup _{\Sigma}|h| \leqq 1$ for all $h \in \Re$. From this it follows easily that $\hat{K}_{0 \Omega}$ containes $\Sigma$.

To prove the theorem, assume that $(E, \varphi)$ does not satisfy $(\Gamma)$, i.e. that the envellope of holomorphy $\hat{K}$ of a compact set $K$ in $E$ contains a set $A$ in the filter defining a boundary point $r$ of $(E, \varphi)$. Then every holomorphic function on $E$ would be bounded on $A \cap E_{0} \neq \phi$, as it is so on $\hat{K}$. Since every function in $\Re$ is holomorphically prolongable to $E$, it would be bounded on $A \cap E_{0}$. Hence, from the above consideration, there exists a compact set $K_{0}$ in $E_{0}$ such that $\hat{K}_{0 \Omega} \supset \Lambda \cap E_{0}$. By the theorem of Cartan-Thullen, ${ }^{21)}$ any function in $\Re$, especially $f_{0}$, is holomorphically prolongable over $r$ to an unramified Riemann domain. Therefore, the existence domain of $f$ would properly contain $(E, \varphi)$. This is a contradiction.

Moreover, we can show that $E_{0}$ coincides with $E$. Assume that there exists a point $x$ in $E-E_{0}$. If $\Delta$ is a compact neighborhood of $x$ in $E$, every function in $\AA$ would be bounded on $\Delta \cap E_{0} \neq \phi$. By the same reason as above $f_{0}$ would be prolongable over $x$ to an unramified Riemann domain. Consequently $\varphi$ would be a local homeomorphism around $x$, which contradicts $x \notin E_{0}$.

Corollary. Let $(E, \varphi)$ be an n-dimensional Riemann domain of holomorphy. If every holomorphic function on $E_{0}$ is holomorphically prolongable to $E,(E, \varphi)$ is unramified.

In partiqular, if the analytic subset $E-E_{0}$ of $E$ is of dimension $\leqq n-2,{ }^{22)}$ the assumption of our Corollary is evidently satisfied.

21) The fundamental theorem in H. Cartan and P. Thullen [1].

22) It is known that in this case $(E, \phi)$ is unramified without any restriction. Cf. $H$. Grauert and Remmert [8]. 


\section{REFERENCES}

[1] H. Cartan and P. Thullen: Zur Theorie der Singularitäten der Funktionen mehrerer komplexer Veränderlichen, Math. Ann. 106 (1932), pp. 617-647.

[2] H. Behnke and P. Thullen: Theorie der Funktionen mehrerer komplexer Veränderlichen (1934).

[3] H. Behnke and K. Stein: Konvergente Folgen von Regularitätsbereichen und die Meromorphiekonvexität, Math. Ann. 116 (1939), pp. 204-216.

[4] H. Cartan: Séminaire de E.N.S. (Paris) 1951-52.

[5] H. Cartan: Séminaire de E.N.S. (Paris) 1953-54.

[6] K. Oka: Sur les fonctions de plusieurs variables complexes. IX. Domaines finis sans point critique intérieur, Jap. J. Math. 23 (1953), pp. 97-155.

[7] H. Grauert: Charakterisierung der holomorph vollständigen komplexen Räume, Math. Ann. 129 (1955), pp. 233-259.

[8] H. Grauert and R. Remmert: Konvexität in der komplexen Analysis, Comment. Math. Helv. 31 (1957), pp. 152-183.

[9] H. Grauert and R. Remmert: Sur les revêtements analytiques des variété analy tiques, Comptes Rendus 245 (1957), pp. 918-921.

[10] R. Iwahashi: Domains spred on a complex space, J. Math. Soc. Japan. Vol 9, No. 4 (1957).

[11] R. Remmert: Holomorphe und meromorphe Abbildungen komplexer R̈̈ume, Math. Ann. 133 (1957).

\section{Mathematical Institute}

Nagoya University 